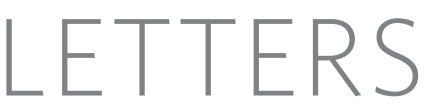

\title{
Molecular basis of RNA-dependent RNA polymerase II activity
}

\author{
Elisabeth Lehmann ${ }^{1}$, Florian Brueckner ${ }^{1} \&$ Patrick Cramer $^{1}$
}

RNA polymerase (Pol) II catalyses DNA-dependent RNA synthesis during gene transcription. There is, however, evidence that Pol II also possesses RNA-dependent RNA polymerase (RdRP) activity. Pol II can use a homopolymeric RNA template, can extend RNA by several nucleotides in the absence of $\mathrm{DNA}^{2}$, and has been implicated in the replication of the RNA genomes of hepatitis delta virus (HDV) ${ }^{3,4}$ and plant viroids ${ }^{5}$. Here we show the intrinsic RdRP activity of Pol II with only pure polymerase, an RNA template-product scaffold and nucleoside triphosphates (NTPs). Crystallography reveals the template-product duplex in the site occupied by the DNA-RNA hybrid during transcription.
RdRP activity resides at the active site used during transcription, but it is slower and less processive than DNA-dependent activity. RdRP activity is also obtained with part of the HDV antigenome. The complex of transcription factor IIS (TFIIS) with Pol II can cleave one HDV strand, create a reactive stem-loop in the hybrid site, and extend the new RNA $3^{\prime}$ end. Short RNA stem-loops with a $5^{\prime}$ extension suffice for activity, but their growth to a critical length apparently impairs processivity. The RdRP activity of Pol II provides a missing link in molecular evolution, because it suggests that Pol II evolved from an ancient replicase that duplicated RNA genomes.

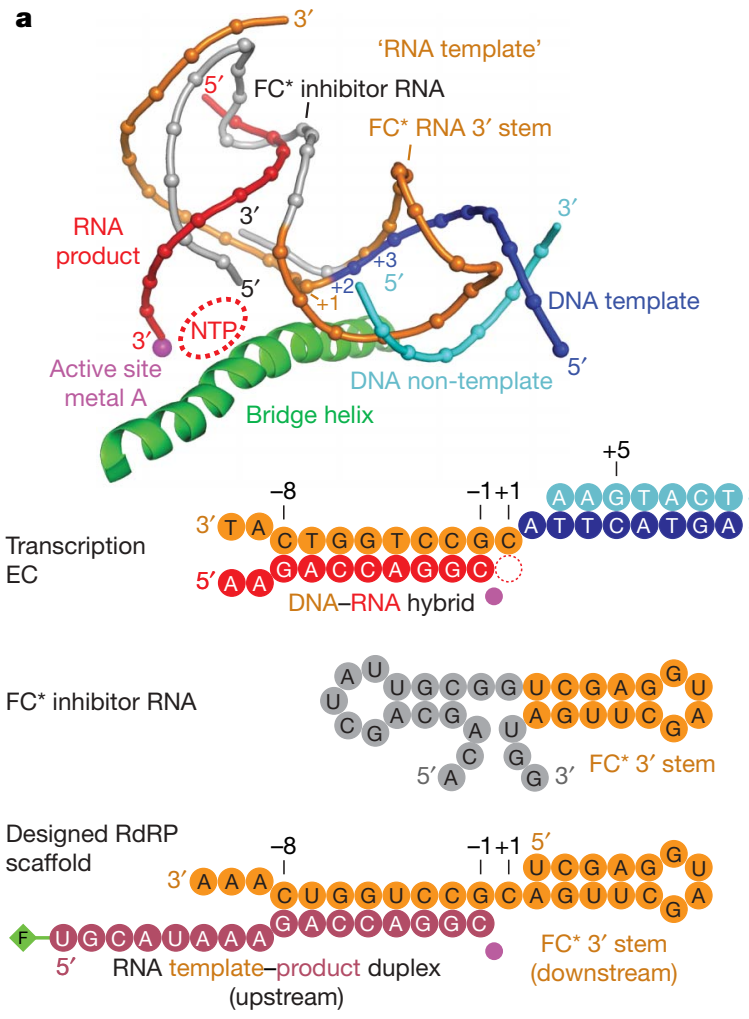

Figure 1 | RNA-dependent RNA polymerase II activity. a, Structure-based design of the RdRP scaffold. Structures of the complete Pol II EC ${ }^{7}$ and the Pol II-FC* RNA inhibitor complex ${ }^{9}$ were superimposed by fitting aspartate loop residues in the active site. The nucleic acids in the active centre of the two complex structures $\left(\mathrm{EC}^{7}\right.$ and $\left.\mathrm{FC}^{*} \mathrm{RNA}^{9}\right)$ are shown. The RdRP scaffold derived by combination of a forked RNA template-product duplex with the $3^{\prime}$ stem of FC* RNA is shown at the bottom. The RNA product strands are in red (transcription EC) or raspberry (RdRP EC), and the RNA template strand is in orange. The FAM fluorescent label is shown as a green diamond. The same colour code is used throughout. The $5^{\prime}$ stem of FC* RNA, which is
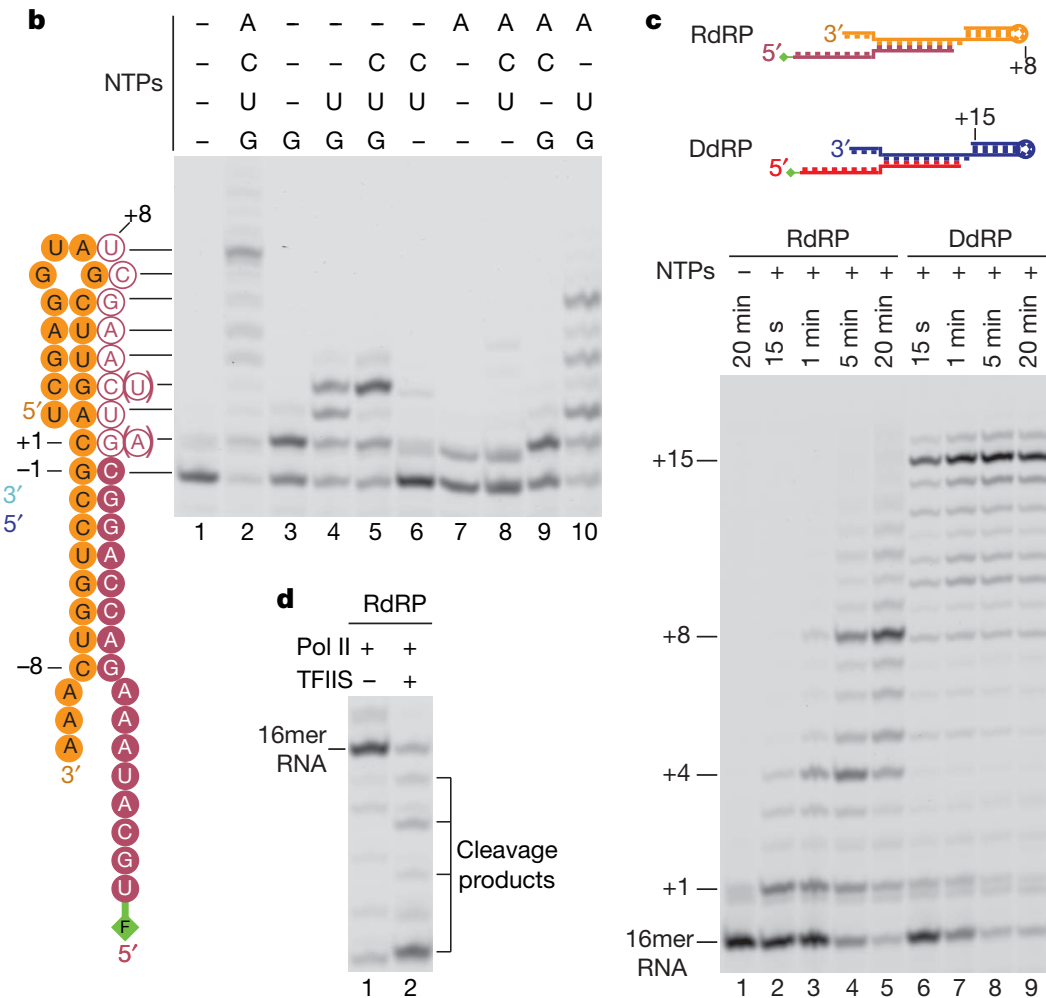

not used here, is in grey. The nucleotide addition site is denoted +1 , and upstream and downstream positions are depicted with negative and positive numbers, respectively. $\mathbf{b}$, RNA synthesis with the RdRP scaffold from a. Lane 1 shows the fluorescently labelled reactant RNA. In lanes 2-10 the RNA scaffold was incubated with pure Pol II and different types of NTP (see Methods). UMP misincorporation at position +3 (lane 4) was confirmed by mass spectrometry (not shown). c, Time courses of RNA synthesis with the RdRP scaffold (lanes 2-5) and the corresponding DNA template-containing scaffold DdRP (lanes 6-9). d, TFIIS-induced 3'-RNA cleavage in the RdRP EC. 
Structural studies of the transcription elongation complex (EC) revealed a B-form DNA duplex entering the downstream polymerase cleft, and a hybrid duplex of DNA template with RNA product above the active $\operatorname{site}^{6-8}$ (Fig. 1a). During RdRP activity, the hybrid site and the downstream cleft are expected to accommodate A-form RNA duplexes. The downstream cleft can accommodate A-RNA, as observed for the $3^{\prime}$ stem of the RNA inhibitor FC* (ref. 9) (Fig. 1a). The FC* $3^{\prime}$ stem overlaps with two DNA template positions downstream of the NTP-binding site (position +1 ; Fig. 1a), suggesting that an RNA template could enter the active site in a similar manner to DNA.

To test whether Pol II can indeed use an RNA template, we prepared an RNA scaffold that combined the FC* $3^{\prime}$ stem with a putative RNA template-product duplex (RdRP scaffold; Fig. 1a). To monitor potential RNA elongation, the product strand was labelled at the $5^{\prime}$ end with 6-carboxyfluorescein (FAM). Incubation of this scaffold with Pol II and NTPs led to RNA elongation (Fig. 1b, lanes 1 and 2), indicating the formation of an active RdRP EC. Most RNA was elongated by eight nucleotides, but shorter and longer products were also observed. Mass spectroscopy of the products was consistent with RNA-templated synthesis (Supplementary Table 1). Incubation of the RdRP EC with subsets of NTPs led to products that generally arose from templated nucleotide addition (Fig. 1b, lanes 3-10), but the absence of the cognate NTP also permitted misincorporation. At position + 1, AMP could be incorporated instead of GMP; at position +3 , UMP could replace CMP. Misincorporation did not result from the use of RNA as a template, because a corresponding DNA template (scaffold DdRP; Fig. 1c) produced the same product pattern (Supplementary Fig. 1). These results show the intrinsic RdRP activity of Pol II.

To unravel the structural basis of the RdRP activity, we determined the crystal structure of the complete Pol II bound to an RdRP scaffold. A scaffold with a 5' extension of six nucleotides sufficed to form an active RdRP EC (Supplementary Fig. 2), and enabled crystallographic analysis at $3.8 \AA$ resolution to be made (Fig. 2, Supplementary Fig. 3, Supplementary Table 2, and Methods). The structure revealed the RNA template-product duplex in the site occupied by the DNA-RNA hybrid during transcription. The RNA product $3^{\prime}$ end was bound to the catalytic metal ion A (Fig. 2). RNA synthesis occurred at the active site used during transcription, because product RNA was cleaved from the $3^{\prime}$ end by TFIIS (Fig. 1d), which stimulates RNA cleavage at the active site in the $\mathrm{EC}^{10}$, and in a Pol II-RNA complex ${ }^{2}$. For positions -1 to -5 , the structure of the RNA duplex was essentially identical to that of the DNA-RNA hybrid ${ }^{7}$; however, at the upstream positions -6 to -9 , changes in phosphate positions of up to $3 \AA$ resulted in a decreased helical rise (Fig. 2b).

To compare Pol II activity on the RdRP scaffold and on a corresponding DNA template scaffold, we conducted a time course experiment (Fig. 1c). Pol II rapidly reached the end of the DNA template but was slower and stopped prematurely on the RNA template (Fig. 1c). This was not due to a different scaffold affinity for Pol II (Supplementary Fig. 4). To investigate the premature stop, we altered the scaffold (Supplementary Fig. 2). When the downstream duplex length was increased or decreased by 1 base pair (bp), the maximum product length changed accordingly (Supplementary Fig. 2b, lanes 3 and 4 ), suggesting that the uracil at position +9 posed an obstacle to elongation. However, synthesis still stopped at position +8 when this uracil was replaced by cytosine or when the downstream duplex was replaced by a single strand (Supplementary Fig. 2b, d). Thus, Pol II could use single-stranded or double-stranded downstream RNA templates and showed impaired processivity, independently of the nature of downstream RNA.

To investigate the physiological significance of the RdRP activity, we studied a terminal segment of the HDV antigenome (Fig. 3a). In cell extracts, this segment directs RNA synthesis that is sensitive to the Pol II inhibitor $\alpha$-amanitin ${ }^{11,12}$. The reaction involved RNA strand cleavage followed by elongation of the new $3^{\prime}$ end ${ }^{11,12}$. The cleaved HDV segment apparently forms an RNA template-product stemloop and a downstream RNA duplex, which we shortened by replacing a natural bulge with a loop (HDV scaffold; Fig. 3a). Incubation of this scaffold with Pol II and NTPs resulted in RNA synthesis up to the end of the template, although synthesis also stopped prematurely (Fig. 3b, lane 8). RNA synthesis was strictly dependent on the template. Only cognate NMPs were incorporated at positions +1 and +2 (Fig. 3b, lanes 2-6). When UTP was omitted from the reaction, RNA synthesis stopped at position +13 as expected (Fig. 3b, lane 7). RNA synthesis was slow, but some final product appeared within $1 \mathrm{~min}$ (Fig. 3d). These in vitro data match the evidence for Pol II-dependent HDV replication in $v i v o^{3,4}$, and argue for the physiological significance of the RdRP activity of Pol II.

The higher processivity of Pol II on the HDV scaffold than on the RdRP scaffold may originate from the different upstream templateproduct duplex, which forms a stem-loop instead of a forked end (Fig. 3c). We therefore analysed two chimaeric scaffolds that recombine the upstream and downstream regions of the two scaffolds
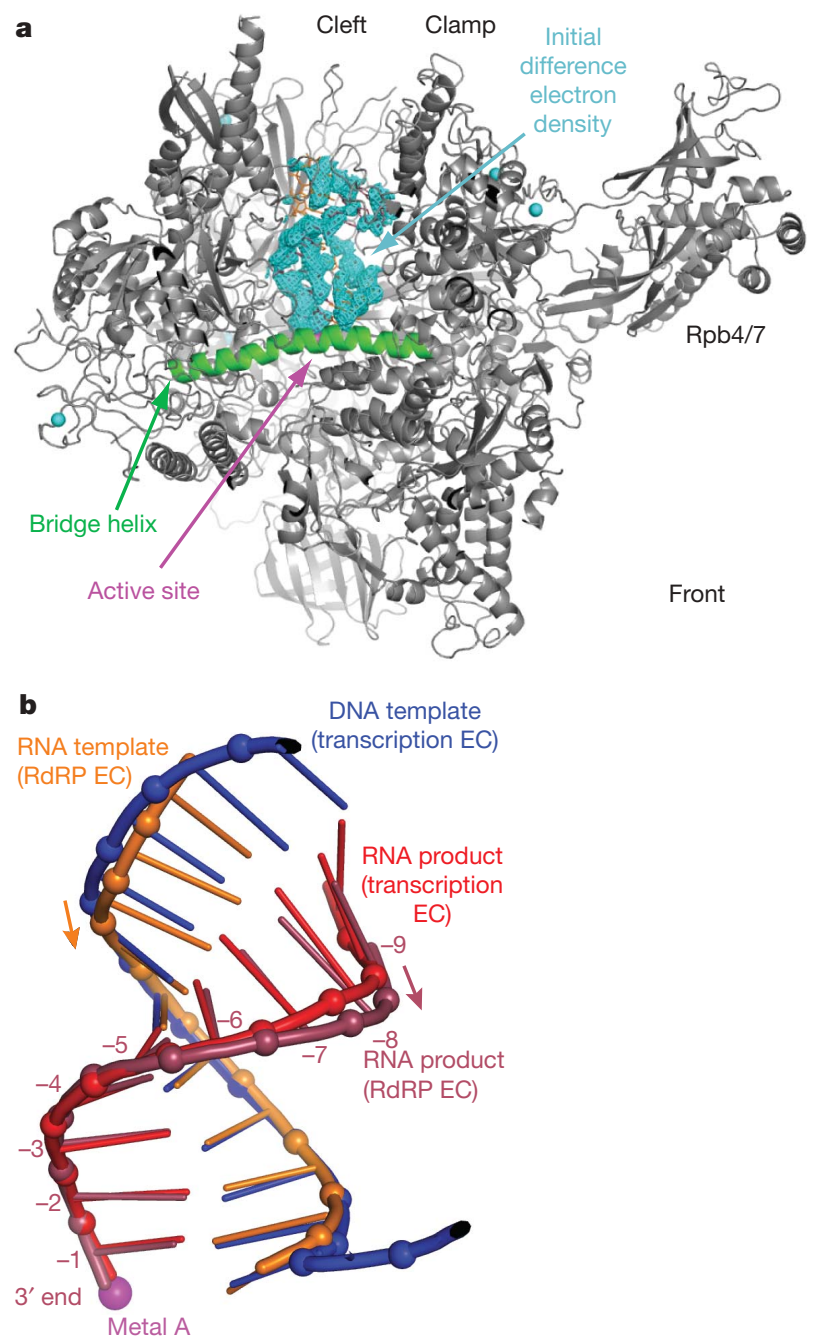

Figure 2 | Crystal structure of a Pol II-RdRP complex. a, Ribbon model of Pol II (grey) with an initial, unbiased difference Fourier electron-density map (cyan, contoured at 2.2 $\sigma$ ). The map was calculated from protein model phases. It reveals the RNA template-product duplex of scaffold RdRP-ss6 (Supplementary Fig. 2) in the active-centre cleft of Pol II. The bridge helix is in green. The catalytic metal ion $\mathrm{A}$ is depicted as a magenta sphere, and $\mathrm{Zn}^{2+}$ ions as cyan spheres. The view is related to that in Fig. 1a by a $90^{\circ}$ rotation around a vertical axis. b, Comparison of the RNA template-product duplex in the RdRP EC with the DNA-RNA hybrid duplex in the transcription EC ${ }^{7}$. Protein structures were superimposed by fitting the active-site aspartate loops. 
(Fig. 3c). Combination of the RdRP upstream region with the HDV downstream region strongly decreased processivity compared with the HDV scaffold (Fig. 3e). In contrast, combination of the HDV upstream stem-loop with the RdRP downstream region enabled run-off synthesis that was not possible with scaffold RdRP (Figs 1c and 3f). Thus, the HDV stem-loop promoted RdRP processivity. Consistently, the stem-loop alone, containing only a two-nucleotide 5 ' extension, formed a functional RdRP EC (Fig. 4a).

To test whether the RdRP-promoting stem-loop can be formed from the HDV terminal segment in vitro, we incubated the segment with pure Pol II-TFIIS complex. This resulted in RNA cleavage at the internal bulge (Fig. 4b). The bulge apparently connected the two RNA duplexes in a flexible way, to enable positioning of the scissile RNA strand at the active site (Fig. 4e). Cleavage produced a 6-bp stem-loop, comparing favourably with the 5-bp stem-loop that forms in extracts ${ }^{11}$. On the addition of NTP, the new $3^{\prime}$ end was elongated up to the end of the template (Fig. 4b, lane 7). Consistently, a corresponding 6-bp stem-loop with a $5^{\prime}$ extension supported RdRP activity (Fig. 4a). Thus, the Pol II-TFIIS complex can cleave the HDV terminal segment and elongate the new $3^{\prime}$ end in vitro, which is consistent with models for HDV replication in vivo.

The 6-bp stem-loop that forms by HDV RNA cleavage in vitro (Fig. 4a) could be structurally revealed in a complex with Pol II (Fig. 4c, d, and Supplementary Table 2). The stem-loop bound the hybrid site, and phosphates at positions -1 to -5 occupied the same locations as in the artificial RdRP EC. However, RNA positions -6 and -7 had an increased helical rise (Fig. 4d), showing that the hybrid site accommodates various RNA duplexes with upstream ends of different structure. The RNA loop was mobile except for one cytidine residue (Fig. 4a, c). Consistently, a double mutation in the loop did not impair activity (Supplementary Fig. 5). Because base-specific Pol II-RNA contacts are absent in both RdRP EC structures, Pol II recognizes the A-RNA stem rather than a particular RNA sequence.

During transcription, upstream template and product strands are separated, whereas the HDV stem-loop probably persists during elongation. Consistently, Pol II readily used stem-loops with 5, 6, 7 or $10 \mathrm{bp}$ as substrates, but not stem-loops with 13,15 or 18 bp (Figs $3 \mathrm{~g}$ and $4 \mathrm{a}$ ). Modelling revealed a clash of the polymerase lid with the RNA stem-loop when it reached a length of 10 or $11 \mathrm{bp}$. The clash may destabilize the RdRP EC and facilitate stalling. This model explains why elongation with the HDV scaffold partly stalled when the stem-loop reached 13-18 bp (Fig. 3a, b), and why replacement of the uracil at the downstream position +8 did not influence stalling (Fig. 3h). The model also explains stalling of the artificial RdRP ECs when a persistent template-product duplex would reach a length of $16 \mathrm{bp}$ (Fig. $1 \mathrm{~b}$ and Supplementary Fig. 2). Similarly, a persistent DNA-RNA hybrid stalls the transcription $\mathrm{EC}^{13-15}$. The limited RdRP processivity in vitro is apparently overcome during HDV replication in vivo by binding of the HDVencoded elongation-stimulatory delta antigen to the polymerase clamp ${ }^{11,12,16}$. Clamp movements and/or RNA repositioning would enable the exit of a persistent stem-loop from the cleft.

Our results also explain the previously observed apparently nontemplated RNA elongation in a Pol II-RNA complex ${ }^{2}$. The RNA used
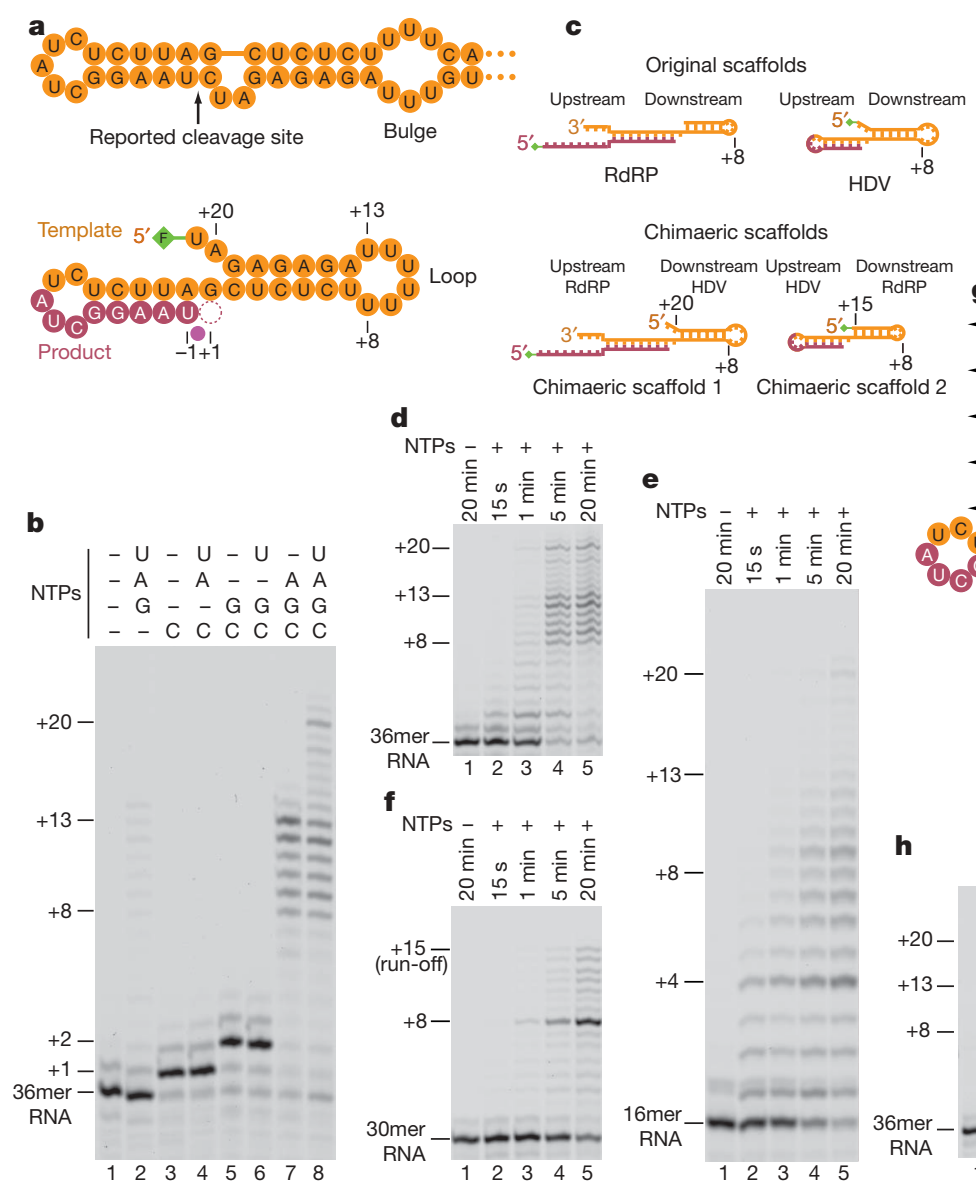

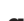
$\stackrel{18 \text {-bp stem }}{\text { 15-bp stem }}$ 13-bp stem ld 2

$13-b p$ stem

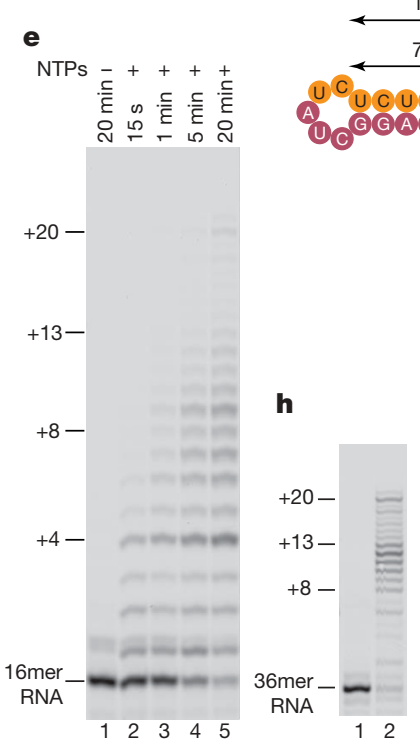

-bp stem
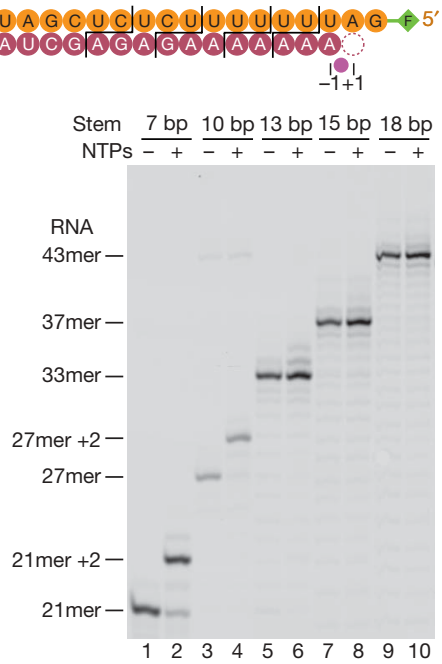

Figure 3 | RNA synthesis with HDV RNA-derived scaffolds a, Schematic representation of the terminal segment of the HDV antigenome (top) and the HDV-derived scaffold (bottom). b, Pol II-dependent RNA synthesis with HDV scaffold. Lane 1 shows the fluorescently labelled reactant RNA. Lanes 2-8 show the elongation products after incubation with various subsets of NTPs as indicated. c, Design of two chimaeric scaffolds that recombine the upstream and downstream regions of the artificial RdRP scaffold and the HDV-derived scaffold. $\mathbf{d}-\mathbf{f}$, Time courses of RNA synthesis with HDV scaffold (d) and with the chimaeric scaffolds 1 (e) and 2 (f). g, HDV-derived stem-loops of different lengths serve as RdRP scaffolds. $\mathbf{h}$, RNA synthesis with an HDV scaffold with $\mathrm{U}$ in position +8 mutated to $\mathrm{C}$. 

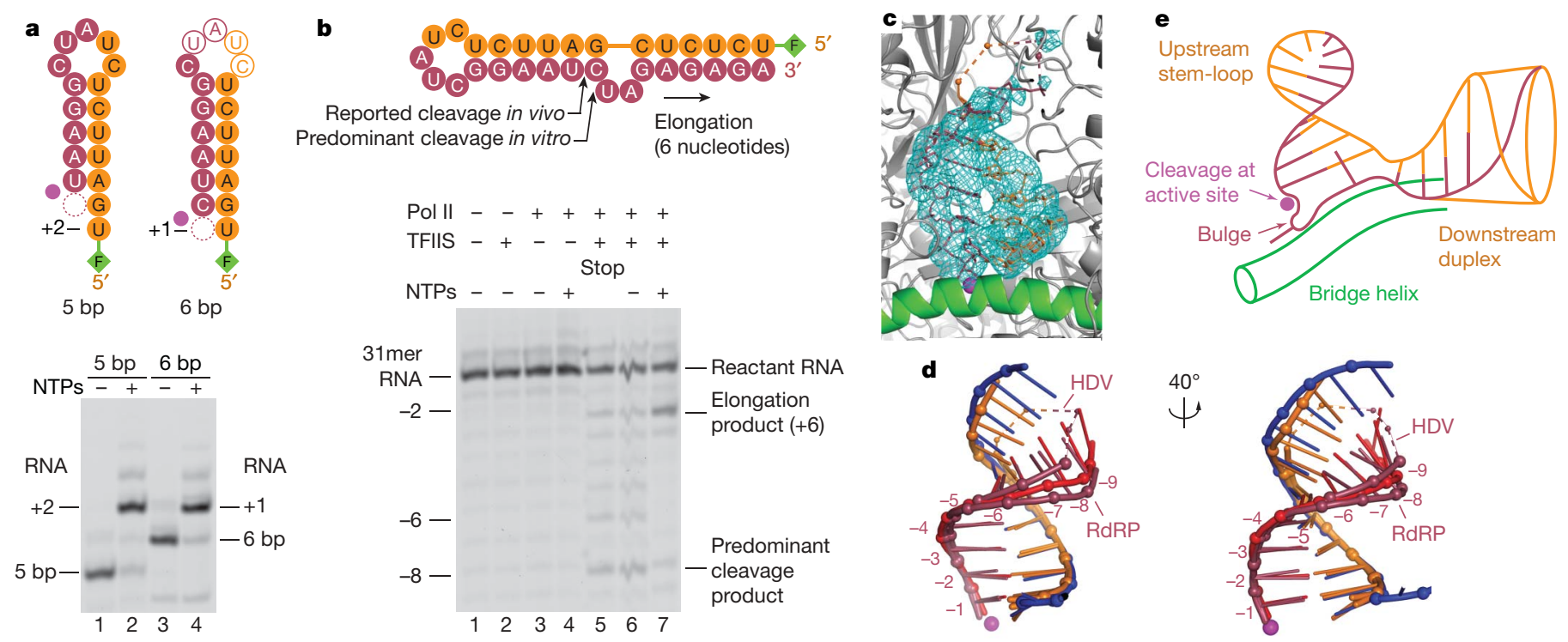

Figure $4 \mid$ Mechanism of HDV replication initiation. a, HDV-derived terminal stem-loops consisting of 5 or $6 \mathrm{bp}$ enable templated incorporation of the next nucleotide(s). $\mathbf{b}$, Pure Pol II-TFIIS complex cleaves the HDV antigenome terminal segment and elongates the newly formed $3^{\prime}$ end on the addition of NTP. For cleavage, Pol II-scaffold complex $(625 \mathrm{nM})$ was incubated with TFIIS $(1.25 \mu \mathrm{M})$ in transcription buffer for $60 \mathrm{~min}$ at $28^{\circ} \mathrm{C}$. For elongation of the cleavage product, the reaction mixture was incubated with $1 \mathrm{mM}$ NTPs at $28^{\circ} \mathrm{C}$ for $20 \mathrm{~min}$. For lane 5 , the cleavage reaction was stopped after $60 \mathrm{~min}$ ('Stop'). c, Difference electron density omit map for the 6-bp HDV stem-loop bound to the hybrid site of Pol II (calculated with

for these studies bound Pol II and was cleaved on the addition of TFIIS; cleavage products were elongated by a few nucleotides ${ }^{2}$. We predict that this RNA formed an 11-bp stem-loop in the hybrid site, and a bulge or a $3^{\prime}$ tail at the active site (Supplementary Fig. 6). TFIISstimulated cleavage then created a new $3^{\prime}$ end at the active site, which was elongated in a templated manner, until a critical templateproduct length was reached. Consistently, an 11-bp stem-loop resembling a cleaved species ${ }^{2}$ supported templated nucleotide incorporation (Supplementary Fig. 6). Our results also explain previously observed cleavage and limited re-extension of a short RNA stem-loop by bacterial RNA polymerase ${ }^{17}$. Thus, earlier observations $^{2,17}$ are apparently examples of the RdRP mechanism described here.

The RdRP activity of Pol II provides a missing link in molecular evolution, because it supports the prediction that an ancestor of Pol II was the enzyme that replicated early RNA genomes ${ }^{18}$. This is supported by the recent findings that the related bacterial RNA polymerase supports not only RNA synthesis with the non-coding $6 \mathrm{~S}$ RNA as template ${ }^{19,20}$ but also RNA-facilitated RNA cleavage ${ }^{21}$. The early RNA replicase apparently evolved to accept DNA as a template during the transition from RNA to DNA genomes. The early replicase was apparently a common ancestor also of distinct single-subunit RdRP enzymes, because these show a Pol II-like core protein fold and active site ${ }^{22}$. It is possible that the ancient RdRP activity of Pol II, which still replicates HDV, is still used in certain cellular processes, because many organisms lack dedicated single-subunit RdRPs. Given the slow nature and the impaired processivity of the RdRP activity in vitro, such processes would require stimulating factors or would be restricted to the generation of smaller RNAs.

\section{METHODS}

Endogenous Saccharomyces cerevisiae core Pol II was purified as described ${ }^{23}$ except that the anion-exchange step was omitted. Recombinant Rpb4/7 and TFIIS were prepared as described ${ }^{10,24}$. Pol II-scaffold complexes were assembled by incubating pure Pol II with two molar equivalents of annealed nucleic acids in transcription buffer as described ${ }^{24}$, at $20^{\circ} \mathrm{C}$ for $30 \mathrm{~min}$. Unless stated otherwise, protein phases only, contoured at $3.0 \sigma$ ). The disordered loop is indicated with a dashed line. The view is as in Fig. 2a. d, Superposition of the RNA template-product duplex in the HDV EC and the RdRP EC (Fig. 2) on the DNA-RNA hybrid duplex in the transcription $\mathrm{EC}^{7}$. Protein structures were superimposed by fitting the active-site aspartate loops. e, Model of initial interaction of the HDV antigenome terminal segment with the Pol II-TFIIS complex. The stem-loop is placed in accordance with the crystal structure (c, d) and the downstream duplex in accordance with the location of the $\mathrm{FC}^{*}$ RNA 3' stem. We predict that the HDV bulge passes the bridge helix and active site, where cleavage occurs.

Pol II-scaffold complex (330 nM) was incubated with NTPs $(1 \mathrm{mM})$ at $28^{\circ} \mathrm{C}$ for $20 \mathrm{~min}$ for RNA extension assays, or with TFIIS ( $660 \mathrm{nM}$ ) for $60 \mathrm{~min}$ for RNA cleavage assays. Reactions were stopped by incubation with an equal volume of $8 \mathrm{M}$ urea, $2 \times \mathrm{TBE}$ (Tris-borate-EDTA) for $5 \mathrm{~min}$ at $95^{\circ} \mathrm{C}$. The FAM-labelled RNA products were separated by denaturing gel electrophoresis ( 1 pmol of RNA per lane, $0.4-\mathrm{mm} 15-20 \%$ polyacrylamide gels containing $7 \mathrm{M}$ urea, at $50-55^{\circ} \mathrm{C}$ ) and revealed with a Typhoon 9400 scanner (GE Healthcare). The crystal structure of the Pol II complex with scaffold RdRP-ss6 (Supplementary Fig. 2) was determined essentially as described ${ }^{7,24}$. Core Pol II was incubated with two molar equivalents of the scaffold and five molar equivalents of Rpb4/7 in $5 \mathrm{mM}$ HEPES $\mathrm{pH} 7.25,40 \mathrm{mM}\left(\mathrm{NH}_{4}\right)_{2} \mathrm{SO}_{4}, 10 \mu \mathrm{M} \mathrm{ZnCl}_{2}, 10 \mathrm{mM}$ dithiothreitol. The complex was purified by size-exclusion chromatography, concentrated to $4 \mathrm{mg} \mathrm{ml}^{-1}$, and crystallized at $22^{\circ} \mathrm{C}$ in hanging drops containing $2 \mu \mathrm{l}$ of sample and $1 \mu \mathrm{l}$ of reservoir solution (200 $\mathrm{mM}$ ammonium acetate, $150 \mathrm{mM}$ magnesium acetate, $50 \mathrm{mM}$ HEPES pH 7.0, 5\% poly(ethylene glycol) 6000, $5 \mathrm{mM}$ TCEP (tris (2-carboxyethyl) phosphine hydrochloride)). The HDV stem-loop was cocrystallized with Pol II under the same conditions. RNA scaffold $(10 \mu \mathrm{M})$ was added to the cryo solutions. For crystallographic details see Supplementary Table 2. Diagrams were prepared with PyMOL (DeLano Scientific).

\section{Received 18 July; accepted 21 September 2007.}

1. Dezelee, S., Sentenac, A. \& Fromageot, P. Role of deoxyribonucleic acid-ribonucleic acid hybrids in eukaryotes. J. Biol. Chem. 249, 5978-5983 (1974).

2. Johnson, T. L. \& Chamberlin, M. J. Complexes of yeast RNA polymerase II and RNA are substrates for TFIIS-induced RNA cleavage. Cell 77, 217-224 (1994).

3. Lai, M. M. C. RNA replication without RNA-dependent RNA polymerase: surprises from hepatitis delta virus. J. Virol. 79, 7951-7958 (2005).

4. Taylor, J. M. Replication of human hepatitis delta virus: recent developments. Trends Microbiol. 11, 185-190 (2003).

5. Rackwitz, H. R., Rohde, W. \& Sanger, H. L. DNA-dependent RNA polymerase II of plant origin transcribes viroid RNA into full-length copies. Nature 291, 297-301 (1981).

6. Gnatt, A. L., Cramer, P., Fu, J., Bushnell, D. A. \& Kornberg, R. D. Structural basis of transcription: an RNA polymerase II elongation complex at $3.3 \AA$ resolution. Science 292, 1876-1882 (2001).

7. Kettenberger, H., Armache, K.-J. \& Cramer, P. Complete RNA polymerase II elongation complex structure and its interactions with NTP and TFIIS. Mol. Cell 16, 955-965 (2004) 
8. Westover, K. D., Bushnell, D. A. \& Kornberg, R. D. Structural basis of transcription: nucleotide selection by rotation in the RNA polymerase II active center. Cell 119, 481-489 (2004).

9. Kettenberger, $\mathrm{H}$. et al. Structure of an RNA polymerase II-RNA inhibitor complex elucidates transcription regulation by noncoding RNAs. Nature Struct. Mol. Biol. 13, 44-48 (2006).

10. Kettenberger, H., Armache, K.-J. \& Cramer, P. Architecture of the RNA polymerase II-TFIIS complex and implications for mRNA cleavage. Cell 114, 347-357 (2003)

11. Filipovska, J. \& Konarska, M. M. Specific HDV RNA-templated transcription by pol II in vitro. RNA 6, 41-54 (2000).

12. Yamaguchi, Y. et al. Stimulation of RNA polymerase II elongation by hepatitis delta antigen. Science 293, 124-127 (2001)

13. Kireeva, M. L., Komissarova, N. \& Kashlev, M. Overextended RNA:DNA hybrid as a negative regulator of RNA polymerase II processivity. J. Mol. Biol. 299, 325-335 (2000).

14. Toulokhonov, I. \& Landick, R. The role of the lid element in transcription by E. coli RNA polymerase. J. Mol. Biol. 361, 644-658 (2006).

15. Naryshkina, T., Kuznedelov, K. \& Severinov, K. The role of the largest RNA polymerase subunit lid element in preventing the formation of extended RNA-DNA hybrid. J. Mol. Biol. 361, 634-643 (2006)

16. Yamaguchi, Y., Mura, T., Chanarat, S., Okamoto, S. \& Handa, H. Hepatitis delta antigen binds to the clamp of RNA polymerase II and affects transcriptional fidelity. Genes Cells 12, 863-875 (2007).

17. Kashlev, M. \& Komissarova, N. Transcription termination: primary intermediates and secondary adducts. J. Biol. Chem. 277, 14501-14508 (2002).

18. Poole, A. M. \& Logan, D. T. Modern mRNA proofreading and repair: clues that the last universal common ancestor possessed and RNA genome? Mol. Biol. Evol. 22, 1444-1455 (2005)
19. Wassarman, K. M. \& Saecker, R. M. Synthesis-mediated release of a small RNA inhibitor of RNA polymerase. Science 314, 1601-1603 (2006).

20. Gildehaus, N., Neusser, T., Wurm, R. \& Wagner, R. Studies on the function of the riboregulator $6 \mathrm{~S}$ RNA from $E$. coli: RNA polymerase binding, inhibition of in vitro transcription and synthesis of RNA-directed de novo transcripts. Nucleic Acids Res. 35, 1885-1896 (2007)

21. Zenkin, N., Yuzenkova, Y. \& Severinov, K. Transcript-assisted transcriptional proofreading. Science 313, 518-520 (2006)

22. Salgado, P. S. et al. The structure of an RNAi polymerase links RNA silencing and transcription. PLoS Biol. 4, e434 (2006)

23. Armache, K.-J., Kettenberger, H. \& Cramer, P. Architecture of the initiationcompetent 12-subunit RNA polymerase II. Proc. Natl Acad. Sci. USA 100, 6964-6968 (2003)

24. Brueckner, F., Hennecke, U., Carell, T. \& Cramer, P. CPD damage recognition by transcribing RNA polymerase II. Science 315, 859-862 (2007).

Supplementary Information is linked to the online version of the paper at www.nature.com/nature.

Acknowledgements We thank U. Hennecke and members of the Cramer laboratory for help, and J. Doudna, K. Förstemann, G. Meister and R. Schroeder for discussions. This work was supported by the Deutsche Forschungsgemeinschaft, the SFB646, the Nanoinitiative Munich, the Elitenetzwerk Bayern, the EU grant 3D repertoire, and the Fonds der Chemischen Industrie.

Author Information The coordinates and structure factors for the RdRP EC and the HDV EC have been deposited in the Protein Data Bank under accession codes 2R92 and 2R93, respectively. Reprints and permissions information is available at www.nature.com/reprints. Correspondence and requests for materials should be addressed to P.C. (cramer@LMB.uni-muenchen.de). 